\begin{tabular}{|c|c|c|}
\hline Beitr. Ent. & Berlin & ISSN 0005-805X \\
\hline $50(2000) 1$ & S. $119-128$ & 11.04 .2000 \\
\hline
\end{tabular}

\title{
Zur taxonomischen Unterscheidung männlicher Mordellistena weisei SCHILSKY und Mordellistena bicoloripilosa ERMISCH
}

\section{(Coleoptera, Mordellidae)}

\author{
Mit 3 Figuren
}

\section{GREgOR SCHMITZ und PETER WAGNER}

\begin{abstract}
Summary
Mordellistena weisei SCHILSKY and M. bicoloripilosa ERMISCH (Col., Mordellidae), stemmining mordellid beetles in Artemisia vulgaris (Asteraceae), have frequently been confused in faunistic studies and not always differentiated in biocoenological investigations. In order to help distinguish the males of the two species, the parameres and the coloration of the head and the profemora are described. An analysis of these features shows that the two species can be distinguished by the shape of their parameres, but not by their coloration. Paramere size did not depend on body size in either species.

\section{Zusammenfassung}

Die in Stengeln von Artemisia vulgaris (Asteraceae) minierenden Mordellistena weisei SCHLSKY und M. bicoloripilosa ERMISCH (Col., Mordellidae) sind bei faunistischen Untersuchungen häufiger verwechselt und bei biozoenotischen Analysen zur Stengelfauna nicht immer differenziert worden. Zur sicheren Unterscheidung der Männchen beider Arten werden die Paramerenform und die Färbungsverteilung an Kopf und Vorderfemura beschrieben. Beide Arten sind über ihre Paramerenform, nicht aber über ihre Färbungsverteilung, eindeutig zuzuordnen. Die Größe ihrer Parameren ist von der Körpergröße unabhängig.
\end{abstract}

Key words

Mordellistena weisei - Mordellistena bicoloripilosa - Mordellidae - taxonomy - genital structure parameres - coloration - variability - Artemisia vulgaris - stem miners.

\section{Einleitung}

Die meisten Mordellistena-Arten (Col., Mordellidae) entwickeln sich in Stengeln mehrjähriger Kräuter, wo sich die Larven vom Mark, gelegentlich aber auch von anderen minierenden oder gallerzeugenden Larven ernähren (KOCH, 1989 bzw. SUMERFORD \& ABRAHAMSON, 1995). Aufgrund der selten praktizierten Zucht aus gesammeltem Pflanzenmaterial (vgl. SCHMITZ, 1995), der geringen ökonomischen Bedeutung (vgl. FRITZSCHE et al., 1968) und der schwierigen Taxonomie sind Angaben zu Brutpflanzen, Lebensraumansprüchen und Verbreitung der Arten unvollständig und wenig abgesichert. Letztlich dürfte auch die zum Aufkleben ungünstige Körperform dazu beigetragen haben, daß die Mordellidae insgesamt, insbesondere aber die in Deutschland 67 Arten umfassende Gattung Mordellistena (KÖHLER \& KLAUSNITZER, 1998), im Gegensatz zu anderen endophytisch sich entwickelnden Coleopteren (Scolytidae, Cerambycidae, Curculionidae) wissenschaftlich vernachlässigt worden sind. 
Kritische Überprüfungen verschiedener Sammlungsbelege aus dem Rheinland brachten bei verschiedenen Arten ein großes Maß an falschen Determinationen zutage (KöHLER 1998). Dafür dürften neben den zu knappen Merkmalsbeschreibungen und den sehr groben Parameren-Abbildungen von ERMISCH (1969) auch das weitgehende Fehlen einer Beschreibung der intraspezifischen Variabilität in der Bestimmungsliteratur verantwortlich sein.

Wie Untersuchungen von SCHMITZ (1996a) zur Phytophagenfauna vom Beifuß, Artemisia vulgaris (Asteraceae), zeigten, betreffen Verwechselungen auch das Artenpaar Mordellistena weisei SCHILSKY, 1895 / M. bicoloripilosa ERMISCH, 1967. Beide Arten gehören innerhalb der Untergattung Mordellistena s. str. zur Gruppe der weisei SCHILSKY, die sich durch eine zweifarbige Behaarung der Elytren auszeichnet (ERMISCH, 1967). M. bicoloripilosa war vor den erwähnten Untersuchungen für das Rheinland nicht angegeben (KÖHLER, 1995), so daß, da auch $M$. brunneospinosa ERMISCH und $M$. flavispinosa HUBTH. noch nicht nachgewiesen sind, $M$. weisei als die einzige im Rheinland vorkommende zweifarbig behaarte Art angesehen wurde (vgl. KoCH, 1968). Die Auswertung der in großer Zahl aus Stengeln von $A$. vulgaris gezogenen Mordellistenen zeigte jedoch, daß $M$. bicoloripilosa sogar deutlich häufiger ist als $M$. weisei (Präsenz: 2-fach; Abundanz: 7-fach) (SCHMrTz, 1995).

Eine sichere Zuordnung der beiden Arten ist nicht nur von faunistischem Interesse, sondern auch von biozoenologischer Bedeutung, da sie wichtige Primärkonsumenten und Parasitoidenwirte im Mikroökosystem Beifußstengel darstellen (DENYS, 1996, SCHMITZ, 1996a). Dieses System kann wiederum zur Untersuchung von Besiedlungsprozessen in anthropogen geprägten Landschaften herangezogen werden (SCHMITZ 1996b, DENYS 1997, DENYS \& SCHMIDT 1998). Darüber hinaus ist eine sichere taxonomische Trennung der beiden syntop vorkommenden Arten für vergleichende Untersuchungen zur Einnischung notwendig $(M$. weisei: vgl. KLAUSNITZER 1967).

Aus den genannten Gründen werden im folgenden die taxonomischen Unterschiede männlicher $M$. weisei und $M$. bicoloripilosa anhand der wichtigsten Unterscheidungsmerkmale, nämlich der Rotfärbung an Kopf und Vorderfemura und der Paramerenform, dargestellt (eine Bearbeitung der Weibchen ist in einem zweiten Schritt beabsichtigt). Die Variabilität dieser Merkmale wird beschrieben, um mögliche Merkmalsüberschneidungen aufzudecken. Darüber hinaus wird getestet, ob die Variabilität in der Paramerengröße auf unterschiedliche Körpergrößen zurückzuführen ist.

\section{Material und Methode}

Das Material stammt im wesentlichen aus Aufsammlungen aus dem Bonner Raum (vgl. SCHMITZ 1996b). Einbezogen wurden auch die Typen von $M$. weisei und $M$. bicoloripilosa und Tiere aus der Sammlung KÖHLER (nur Sichtung und Messung).

Die Parameren wurden (z. T. nach Mazeration des Hinterleibendes in kochender, 10\%iger $\mathrm{KOH}$ ) herauspäpariert und mit ihren Dorsalseiten (vgl. Schema in Fig. 2) auf feste Klarsichtfoliekärtchen geklebt, was eine beidseitige Betrachtung ermöglicht (Stereolupe bis 100-fach und Mikroskop bis 400-fach).

Folgende Anmerkungen zur Lagebeziehung der Parameren seien vorangeschickt: Im allgemeinen Teil zum Bestimmungschlüssel der Mordellidae übernimmt ERMISCH (1969) von FrANCISCOLO eine Abbildung [als ,Fig. 4"] zum männlichen Genitalapparat der Familie. Die von ERMISCH gesetzte Beschriftung ,linke“ bzw. ,rechte Paramere“ entspricht in ihrer Seitenzuordnung nicht der heute allgemein gültigen Konvention (z. B. SNODGRASS, 1993).

Im Text des Bestimmungsschlüssels stimmt er jedoch mit dieser überein (z. B. Punkt 45, S. 181). Besondere Verwirrung ist zusätzlich dadurch gegeben, daß ERMISCH in den zugehörigen Zeichnungen das rechte Paramer linksseitig und das linke rechtsseitig darstellt, wodurch die 
Anordnung nicht mehr der natürlichen Lagebeziehung entspricht. Um die Vergleichbarkeit mit den Figuren von ERMISCH (1969 und andere Arbeiten) zu vereinfachen, folgen wir (in Fig. 24) trotz der genannten Ungereimtheiten seiner Art der zeichnerischen Darstellung.

Die Längenabmessung der Parameren erfolgte von der Basis bis zur Spitze des Dorsalastes ( $=$ mit Trichomen versehener Ast) bei 100-facher Vergrößerung (Meßgenauigkeit $10 \mu \mathrm{m}$ ). Die Elytren wurden bei 25-facher Vergrößerung (Meßgenauigkeit $0,1 \mathrm{~mm}$ ) vom Hinterende bis zum Vorderrand der Schulterbeule vermessen (vorderer Elytrenrand ist bei präparierten Tieren oft vom Pronotumhinterrand verdeckt). Zur Berechung des biometrischen Zusammenhangs wurden die Korrelationskoeffizienten mittels SPSS 8.0 errechnet.

\section{Ergebnis}

\subsection{Rotfärbung an Kopf und Vorderfermura}

Die rötliche Aufhellung der Männchen beider Arten ist, wie bei anderen Arten der Gattung, in der Regel ausgedehnter und intensiver als die der artgleichen Weibchen. Das Farbspektrum reicht von braun über rostrot bis gelborange und zeigt sich vor allem an den Vorder-bis Mittelbeinen und Teilen des Kopfes (Postclypeus, Anteclypeus, Labrum, basale Teile von Antennen, Mandibeln und Palpen).

Die Rotfärbung ist bei $M$. bicoloripilosa ausgedehnter als bei $M$. weisei (Fig. 1, Tab. 1). Letztere Art zeichnet sich durch eine größere Variabilität in der Färbung aus. Die sicherste Trennung der Männchen beider Arten nach äußeren Merkmalen ist anhand der Färbung des Clypeus möglich, dessen zentraler Bereich bei $M$. bicoloripilosa rostrot bis rotorange und bei $M$. weise i in der Regel schwarz ist und einen braunen Rand besitzt. Bei hellen $M$. weisei jedoch (4 von 25 untersuchten Individien) kann der Clypeus rotbraun aufgehellt sein. In diesem Falle ist die Farbabgrenzung zur Frons hin gerade oder konkav (bei $M$. bicoloripilosa konvex). Relativ deutlich sind noch die Unterschiede in der Färbung des sichtbaren Teils der Mandibeln und der 2. und 3. Antennenglieder (bei M. bicoloripilosa intensiver rot als bei $M$. weisei).

Tab. 1: Ausdehnung der Rotfärbung am Vorderkörper von Mordellistena weisei- und M. bicoloripilosaMännchen.

\begin{tabular}{|c|c|c|}
\hline & M. weisei & M. bicoloripilosa \\
\hline Antennen & $\begin{array}{l}\text { braune bis rotbraune Färbung endet am } 2 \text {. } \\
\text { bis } 5 \text {. Glied; selten } 1 \text {. bis } 4 \text {. Glied rostrot } \\
\text { bis rotorange }\end{array}$ & $\begin{array}{l}\text { braune bis rotorange Färbung endet } \\
\text { am } 4 \text {. oder } 5 \text {. Glied, 1. Glied kann } \\
\text { abgedunkelt sein (braun) }\end{array}$ \\
\hline Clypeus & $\begin{array}{l}\text { meist schwarz und am unteren Rand leicht } \\
\text { gebräunt; wenn rotbraune Aufhellung, } \\
\text { dann mit gerader oder konkaver oberer } \\
\text { Farbabgrenzung }\end{array}$ & $\begin{array}{l}\text { meist zentral ausgedehnt rotorange } \\
\text { bis rostrot, Umrandung braun; obere } \\
\text { Farbabgrenzung konvex }\end{array}$ \\
\hline Anteclypeus & schwarz bis rostrot (selten rotorange) & braun bis rotorange \\
\hline Labrum & $\begin{array}{l}\text { meist schwarz mit dunkelbraunen Seiten } \\
\text { und/oder Vorderrand; selten einheitlich } \\
\text { braun }\end{array}$ & $\begin{array}{l}\text { einheitlich rotbraun oder im Zen- } \\
\text { trum braun und randlich rostrot }\end{array}$ \\
\hline Mandibeln & einheitlich schwarz oder basal bis rostrot & braun, basal bis gelborange \\
\hline Maxillarpalpen & $\begin{array}{l}\text { meist 1. und } 2 \text {. Glied, z.T. auch die basale } \\
\text { Hälfte des } 3 \text {. Gliedes gelborange, selten } \\
\text { schwarzbraun }\end{array}$ & $\begin{array}{l}\text { bis mindestens zur Hälfte des } 3 \text {. } \\
\text { Gliedes braun bis gelborange }\end{array}$ \\
\hline Vorderfemura & schwarz bis gelborange & braun bis gelborange \\
\hline
\end{tabular}


Überschneidungen der Färbungsmerkmale finden sich - aufgnund der großen Variabilität von M. weisei - bei den Maxillarpalpen, Antennen und Vorderfemura. Auch der Grad der Rotfärbung am Anteclypeus, den ERMISCH (1967) als wichtiges Merkmal herausstellt, kann bei beiden Arten sehr ähnlich sein.

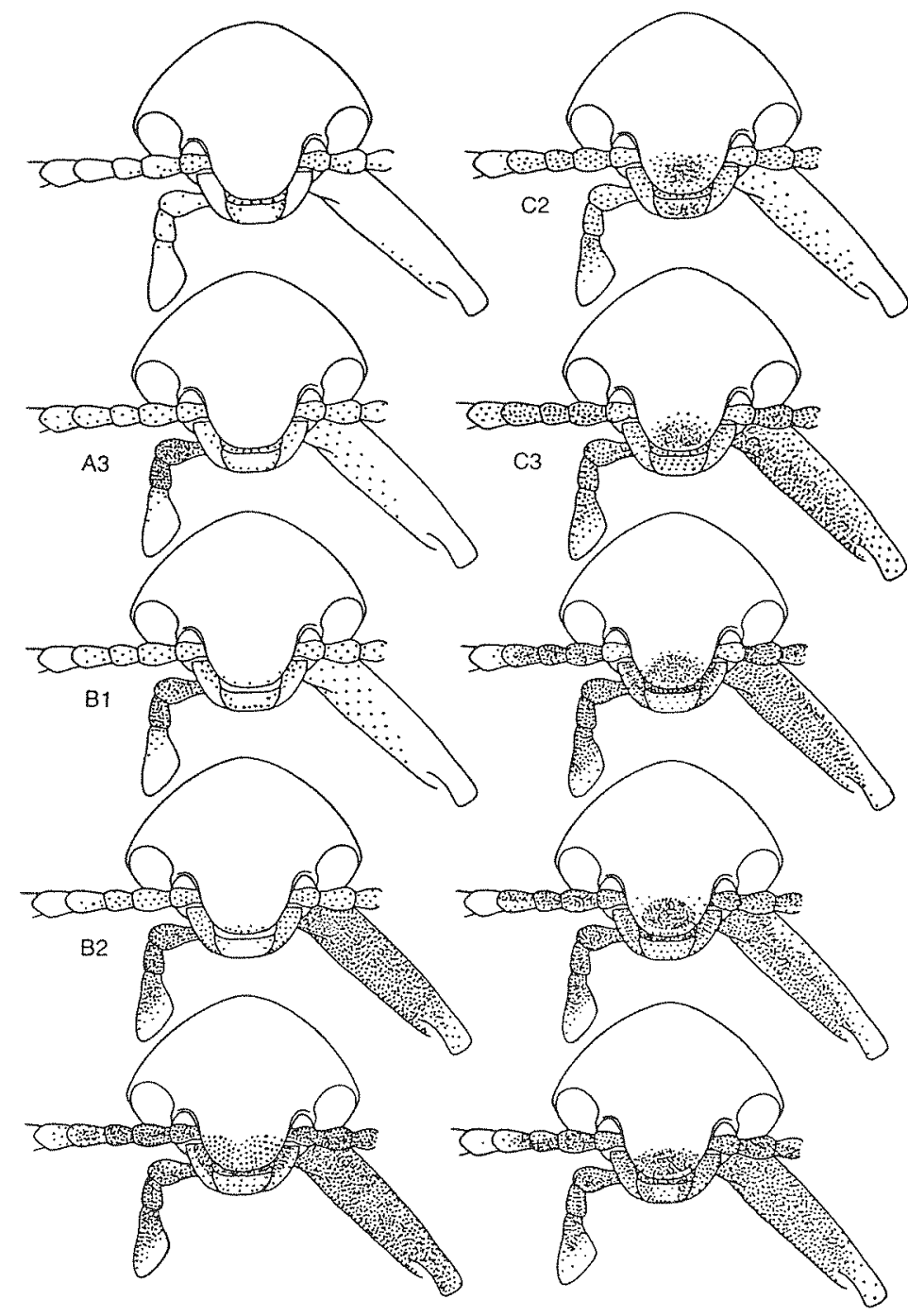

M. weisei

M. bicoloripilosa
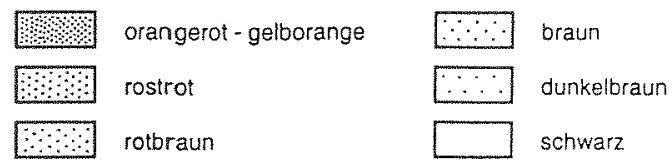

Fig. 1: Ausmaß der Rotfärbung an Kopf (ohne Labialpalpen) und Vorderfemura männlicher Mordellistena weisei und $M$. bicoloripilosa. Die Anhänge sind parallel zur Papierebene dargestellt. Der Typus von $M$. weisei entspricht Individuum B2, der von M. bicoloripilosa dem hellsten Exemplar (vgl. Fig. 3). 


\subsection{Parameren}

Jeweils typische Parameren beider Arten, darunter die des Typus von M. bicoloripilosa, sind in Fig. 2 gegenübergestellt. Zur Dokumentation der Variationsbreite sind je Art neun weitere Paramerenpaare abgebildet (Fig. 3). Basierend auf diesen und anderen, nicht zeichnerisch dokumentierten Parameren ergeben sich die in Tab. 2 aufgeführten Merkmalsunterschiede.

Tab. 2: Merkmalsvergleich der Parameren von Mordellistena weisei und M. bicoloripilosa.

\begin{tabular}{|c|c|c|c|}
\hline \multicolumn{2}{|c|}{ Linkes Parameter } & \multirow{2}{*}{\multicolumn{2}{|c|}{$\begin{array}{l}\text { M. weisei } \\
\begin{array}{l}\text { M. bicoloripilosa } \\
\text { ohne Borsten, Grat und seitlichen Zahn; nicht hohlkehlig; } \\
\text { Spitze aufgebogen* }\end{array}\end{array}$}} \\
\hline \multirow[t]{3}{*}{$\begin{array}{l}\text { allgemeine } \\
\text { Merkmale }\end{array}$} & Ventralast & & \\
\hline & Dorsalast & \multicolumn{2}{|c|}{$\begin{array}{l}\text { Spitze aufgebogen* } \\
\text { mit Borsten (stets distal innen und basal außen); meist mit } \\
\text { Grat, auf dem oft ein Zahn oder Höcker liegt; innen meist } \\
\text { hohlkehlig }\end{array}$} \\
\hline & Basis & \multicolumn{2}{|c|}{$\begin{array}{l}\text { von der Längsachse der Paramerenäste nach außen leicht ab- } \\
\text { gewinkelt; basal des Ventralastes mit zwei Gelenkhöckern }\end{array}$} \\
\hline \multirow[t]{2}{*}{ Ventralast } & Form & $\begin{array}{l}\text { gerade oder höchstens } \\
\text { leicht gekrümmt**, etwas } \\
\text { abgeflacht }\end{array}$ & $\begin{array}{l}\text { meist gleichmäßig gekrümmt**, } \\
\pm \text { stielrund }\end{array}$ \\
\hline & $\begin{array}{l}\text { Länge relativ } \\
\text { zum Dorsalast }\end{array}$ & etwa gleich lang & deutlich $(1 / 3$ bis $1 / 6)$ kürzer \\
\hline \multirow[t]{3}{*}{ Dorsalast } & Spitze & $\begin{array}{l}\text { verjüngt, leicht kapuzen- } \\
\text { förmig }\end{array}$ & breit, deutlich kapuzenförmig \\
\hline & Lage des Grates* & meist mittig & meist nach innen** verlagert \\
\hline & Höcker / Zahn & $\begin{array}{l}\text { flacher Höcker, meist auf } \\
\text { mittlerer Länge des Astes }\end{array}$ & $\begin{array}{l}\text { deutlicher Zahn, meist distal der } \\
\text { Mitte des Astes }\end{array}$ \\
\hline
\end{tabular}

\begin{tabular}{|c|c|c|c|}
\hline \multicolumn{2}{|c|}{ Rechtes Paramer } & \multirow{2}{*}{\multicolumn{2}{|c|}{$\frac{\text { M. weisei }}{\text { M. bicoloripilosa }}$}} \\
\hline \multirow{3}{*}{$\begin{array}{l}\text { allgemeine } \\
\text { Merkmale }\end{array}$} & Ventralast & & \\
\hline & Dorsalast & \multicolumn{2}{|c|}{$\begin{array}{l}\text { mit Borsten (stets distal innen** und basal außen); meist mit } \\
\text { Grat; innen** meist hohlkehlig bis löffelartig }\end{array}$} \\
\hline & Basis & \multicolumn{2}{|c|}{$\begin{array}{l}\text { Achse fast parallel zur Längsachse der Paramerenäste; basal } \\
\text { des Ventralastes mit zwei Gelenkhöckern }\end{array}$} \\
\hline \multirow[t]{3}{*}{ Ventralast } & Form & $\begin{array}{l}\text { leicht gekrümm***, spatel- } \\
\text { förmig, in der Längsachse } \\
\text { leicht gedreht }\end{array}$ & $\begin{array}{l}\text { deutlicher gekrümmt**, } \\
\text { eher stielförmig; aufgebogen* }\end{array}$ \\
\hline & Zahn & $\begin{array}{l}\text { am inneren**, basalen } \\
\text { Rand }\end{array}$ & fehlt \\
\hline & $\begin{array}{l}\text { Länge relativ } \\
\text { zum Dorsalast }\end{array}$ & deutlich ( $1 / 3$ bis $1 / 4)$ länger & etwa gleich lang \\
\hline Dorsalast & & \multicolumn{2}{|c|}{ keine wesentlichen Unterschiede } \\
\hline
\end{tabular}

* zum Betrachter hin, ** zur Längsachse des Paramers

Die auffälligsten Unterschiede zwischen beiden Arten liegen in den relativen Längen der Paramerenäste zueinander und dem Vorhandensein und der Lage eines Zahnes bzw. Höckers: Bei $M$. weisei ist das rechte, bei $M$. bicoloripilosa das linke Paramer das jeweils längere. $M$. weisei zeigt im Gegensatz zu $M$. bicoloripilosa am rechten Paramer einen deutlich längeren ventralen Ast, der auf seiner Innenseite basal bezahnt ist. Verlängert und deutlich bezahnt ist dagegen der dorsale Ast des linken Paramers von $M$. bicoloripilosa, während der von $M$. weisei etwa so lang ist wie der ventrale Ast und nur einen schwachen, weiter basal liegenden Höcker aufweist. 

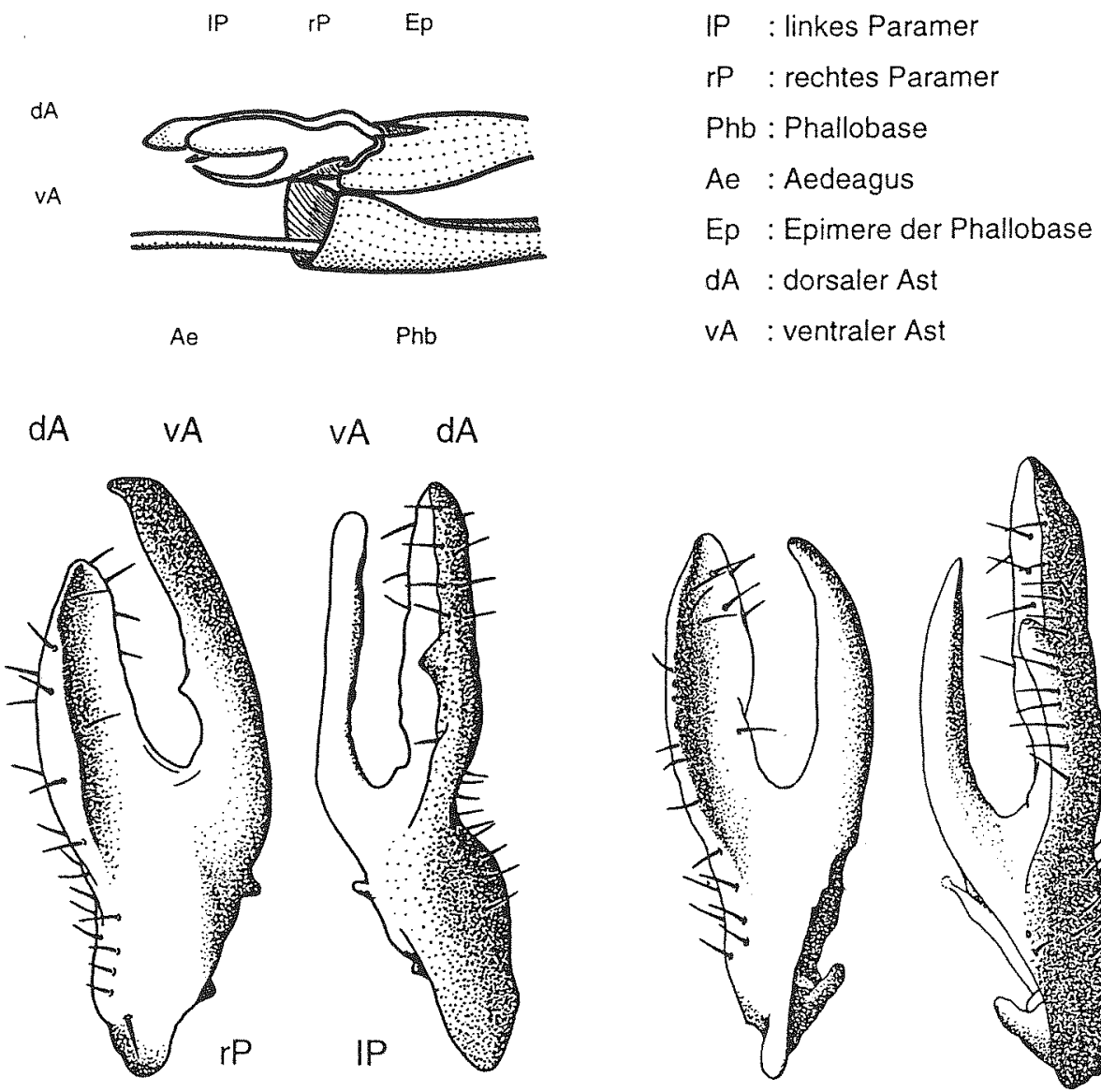

M. weisei
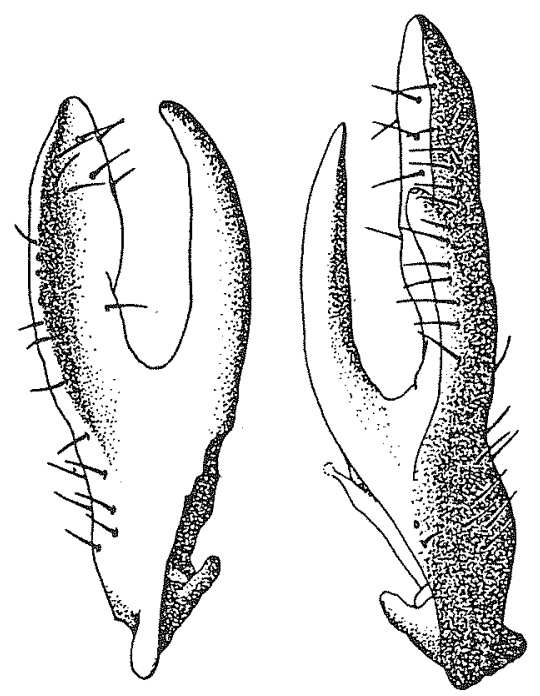

Fig. 2: Ventrale Sicht auf typische Parameren von Mordellistena weisei (Bonn Endenich, 1993; Typus nicht dargestellt, da linkes Paramer beschädigt ist) und M. bicoloripilosa (Typus: AU: Karawanken, 1965), nebst Übersicht zur Lagebeziehung der Parameren.

Bei beiden Arten zeigt sich eine auffallige innerartliche Variabilität in der Gestalt vor allem des linken Paramers. So fehlt den mit $\mathrm{C} 2$ und C3 bezeichneten linken Parameren von $M$. weisei ein Grat auf dem Dorsalast. Bei M. bicoloripilosa weichen die linken Parameren der Tiere C1 und $\mathrm{C} 2$ durch die Ausformung und Lage der Zähne ab.

Die Gesamtlänge der Parameren beider Arten ist nicht mit der Körpergröße (Elytrenlänge) korreliert: $M$. bicoloripilosa $(\mathrm{n}=26)$, linkes Paramer $r^{2}=0,003, \mathrm{p}=0,781$, rechtes Paramer $\mathrm{r}^{2}=$ $0,008, p=0,665 ;$ M. weisei $(n=24)$, linkes Paramer $r^{2}=0,046, p=0,317$, rechtes Paramer $r^{2}=$ $0,144, p=0,067$. 


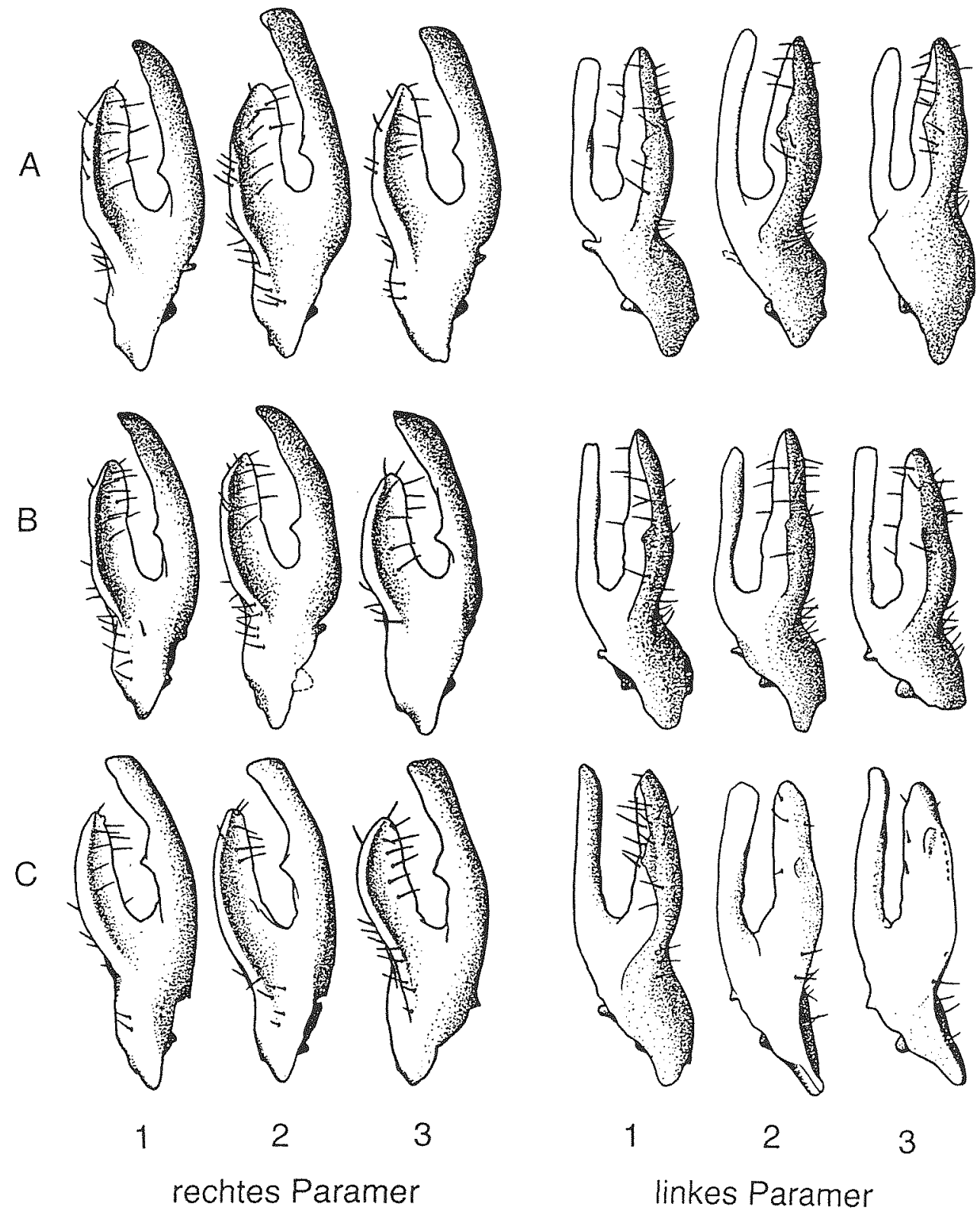

Fig. 3: Linke und rechte Parameren: a) von neun Mordellistena weisei ( $11=\mathrm{Bonn}$ Tannenbusch; $\mathrm{A} 2=$ Bonn Endenich; A3, B1-3 = Bonn Buschdorf; C1-3 = Kripp / Remagen), b) von neun Mordellistena bicoloripilosa (Al-3, B1-3, Cl = Bonn Innenstadt; $\mathrm{C} 2-3=$ Bonn Endenich). 

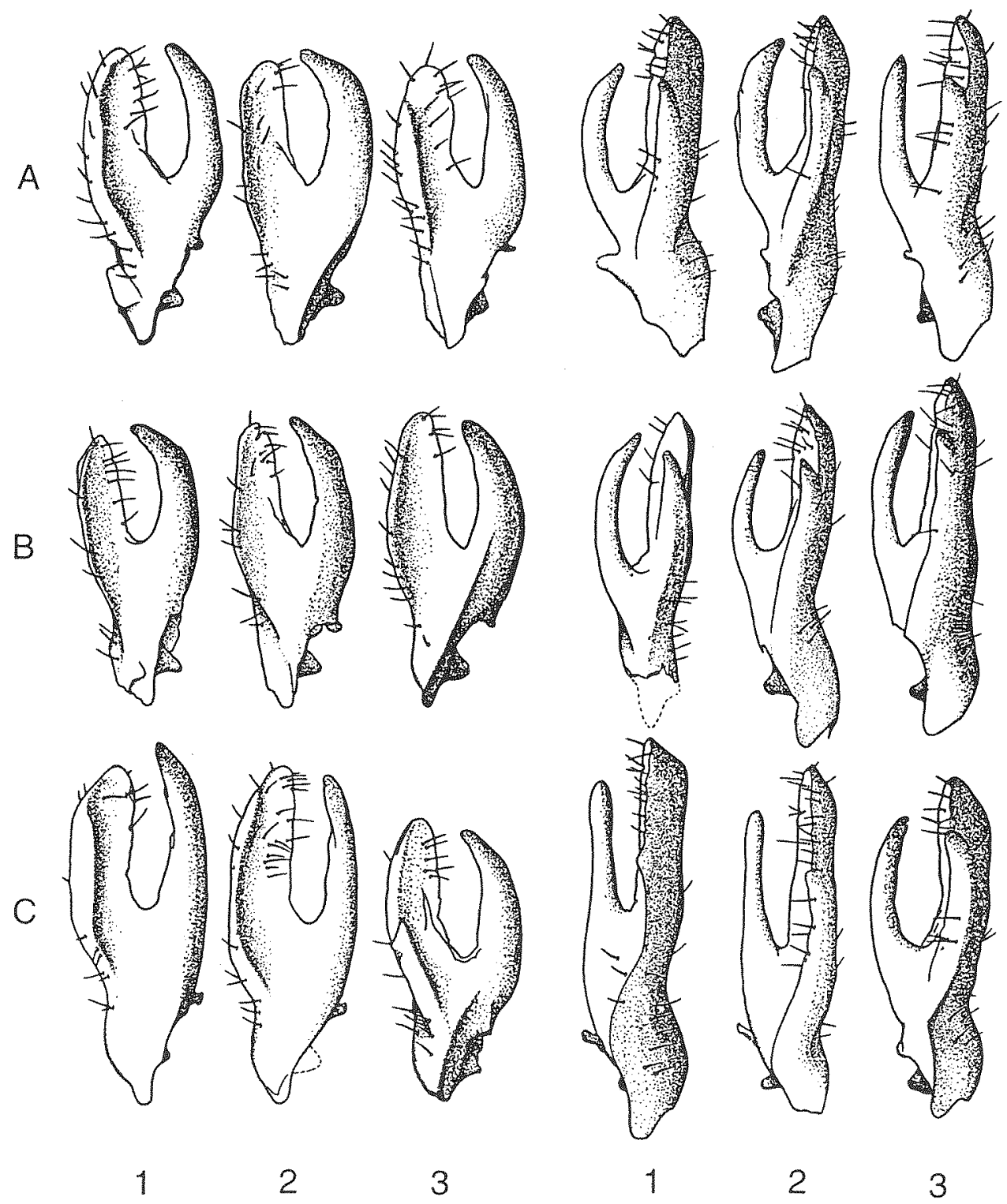

rechtes Paramer
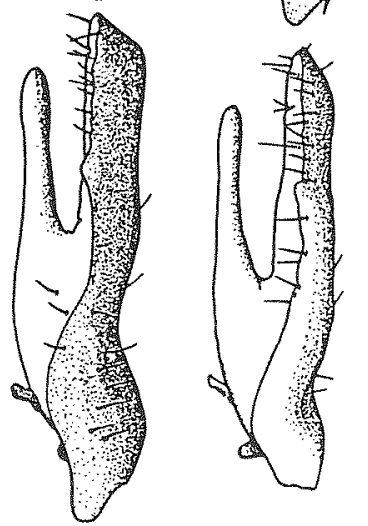

2

1

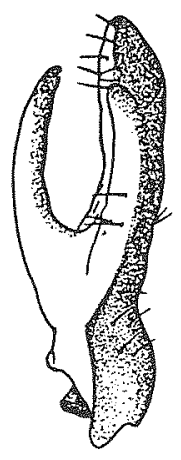

3

linkes Paramer

\section{Diskussion}

Die von ERMISCH (1969) angegebenen Färbungsmerkmale müssen erweitert werden, da beide Arten hierin erheblich variieren. So zeigen sehr stark aufgehellte Exemplare von $M$. weisei und dunkle Exemplare von M. bicoloripilosa große Ähnlichkeiten. Eine Unterscheidung allein anhand der Färbungsmerkmale ist nicht sicher möglich. Hat man sich aufgrund der schlanken 
Gestalt und des lang ausgezogenen Pygidiums davon überzeugt, daß es sich um ein Männchen handelt, kann man mit einiger Sicherheit sehr dunkle Tiere $M$. weisei, sehr helle (aber voll sklerotisierte) Tiere $M$. bicoloripilosa zuordnen.

Die taxonomische Trennung beider Arten ist über Paramerenmerkmale leicht möglich. Abweichende Formen linker Parameren kommen bei beiden Arten vor. Daß es sich hier um Exemplare weiterer, bisher unbeschriebener Arten handelt, läßt sich anhand äußerer Merkmale nicht bestätigen.

Die Variabilität der Parameren läßt sich für beide Arten nicht mit der unterschiedlichen Größe der Tiere erklären. Damit sind $M$. weisei und $M$. bicoloripilosa weitere Beispiele für den von EBERHARD et al. (1998) dargestellten Sachverhalt, daß die Größe männlicher Genitalien weniger von der Körpergröße abhängt als andere, nicht im Dienst der Fortpflanzung stehende Körperteile.

Warum $M$. bicoloripilosa im Rheinland erst in den neunziger Jahren nachgewiesen wurde, obwohl sie hier in Beifußstengeln sehr häufig ist, kann nicht befriedigend beantwortet werden. Entweder liegt es an der Seltenheit, mit der Genitalpräparationen durchgeführt werden, daß die auch früher schon präsente Art stets für $M$. weisei gehalten wurde oder $M$. bicoloripilosa ist erst in neuerer Zeit ins Rheinland (aus dem Osten) eingedrungen. Die Prüfung alter Sammlungsbelege wäre daher anzustreben.

\section{Danksagung}

Wir danken insbesondere Herrn F. KÖHLER (Bornheim) für die Bereitstellung von Exemplaren und für Anmerkungen zum Manuskript sowie Herrn Dr. K. REINHOLD (Bonn) für die Hilfe bei der statistischen Auswertung.

\section{Literatur}

DENYS, C. 1996: Die Insekten-Lebensgemeinschaften in den Stengeln von Artemisia vulgaris L. und deren Artenvielfalt an verschiedenen Standorten (Asteraceae, Anthemideae). - Verh. 14. Internat, Symp. Entomofaunistik Mitteleuropa (SIEEC) München (4.-9.9.94): 321-330.

DENYS, C. 1997: Fördern Ackerrandstreifen die Artendiversität in einer ausgeräumten Agrarlandschaft? Untersuchungen am Beispiel der Insektenlebensgemeinschaft am Beifuß (Artemisia vulgaris L.). Mitt. Dtsch. Ges. Allg. Angew. Ent. 11: 69-72.

DENYS, C. \& SCHMIDT, H. 1998: Insect communities on experimental mugwort (Artemisia vulgaris L.) plots along an urban gradient. - Oecologia 113 (2): 269-277.

Eberhard, W. G.; Huber, B. A.; Rodriguez R. L.; BRICeÑo, R. D.; SAlas, I. \& Rordriguez V. 1998: One size fits all? Relationships between the size and degree of variation in genitalia and other body parts in twenty species of insects and spiders. - Evolution 52 (2): 415-431.

ERMISCH, K. 1967: Neue Mordellistena-Arten in Mitteleuropa und der Balkanhalbinsel (50. Beitrag zur Kenntnis der Mordelliden). - Ent. B1. 63 (2): 110-119.

ERMISCH, K. 1969: Fam. Mordellidae. In: FreUde, H.; HARdE, K. W. \& LoHSE, G. A. (Hrsg.): Die Käfer Mitteleuropas. Bd. 8: 160-196.

FRITZSCHE, R.; GEILER, H. \& SEDLAG, U. 1968: Angewandte Entomologie. - Stuttgart: G. Fischer Verl. $778 \mathrm{~S}$.

KLAUSNITZER, B. 1967: Zur Biologie von Mordellistena weisei SCHILSKY (Col., Mordellidae). - Dtsch. Ent. Z. (N.F.) 5: 477-480.

KocH, K. 1968: Käferfauna der Rheinprovinz. - Decheniana Beih. 13: 1-382.

KOCH, K. 1989: Die Käfer Mitteleuropas - Ökologie. Bd. 2. - Krefeld: Goecke \& Evers. - 382 S.

KöHLER, F. 1995: Anmerkungen zur Käferfauna der Rheinprovinz VIII. Bermerkenswerte Neu- und Wiederfunde (Ins., Col.). - Mitt. Arb.gem. Rhein. Koleopterologen (Bonn) 5: 93-104. 
KöHLER, F. 1998: Anmerkungen zur Käferfauna der Rheinprovinz XII. - Mitt. Arb.gem. Rhein. Koleopterologen (Bonn) 8: 35-51.

KÖHLER, F. \& KLAUSNITZER, B. 1998: Verzeichnis der Käfer Deutschlands (Entomofauna Germanica). Ent. Nachr. Ber., Beih. 4: 1-185.

SCHMITZ, G. 1995: Stengeleklektor-Techniken und ihre Bedeutung für den Nachweis von Stachelkäfern (Col. Mordellidae), - Mitt. Arb.gem. Rhein. Koleopterologen (Bonn) 5: 205-213.

SCHMTTZ, G. 1996a: Urban ruderal sites as secondary habitats for phytophagous insects. - Verh. Ges. Ökol. 26: 581-585.

SCHMITZ, G. 1996b: Phytophagenkomplexe von Artemisia vulgaris L. und Tanacetum vulgare L. (Asteraceae) und deren Beeinflussung durch zunehmende Urbanität der Standorte. - Diss. Univers. Bonn $189 \mathrm{~S}$.

SNOdGrass, R. E. 1993: Principles of insect morphology. - Ithaca, London: Cornell Univ. Press, 667 S.

Sumerford, D. V. \& ABrahamson, W. G. 1995: Geographic and host species effects in Eurosta solidaginis (Diptera, Tephritidae) mortality. - Environ. Ent. 24 (3): 657-662.

\section{Anschrift der Verfasser:}

Dr. GREGOR SCHMITZ

Institut für Evolutionsbiologie und Ökologie

An der Immenburg 1

D - 53121 Bonn

Deutschland
PETER WAgner

Rudolf-Hahn-Straße 21

D - 53225 Bonn

Deutschland

\section{Besprechungen}

HALL, J. P. W.: A Revision of the Genus Theope, its Systematics and Biology (Lepidoptera: Riodinidae: Nymphidiini), - Gainesville: Scientific Publishers, 1999. - viii + 128 p., 274 figs. - ISBN 0-945417-95-0.

Der Autor legt mit dieser Bearbeitung das Ergebnis einer über fünfjährigen intensiven Forschung über diese neotropische Gattung vor. Diese umfaßt gegenwärtig 68 valide Arten, die in 14 Artengruppen eingeordnet werden.

Nach einleitenden Bemerkungen werden im Kapitel Taxonomy Ausführungen zur Charakterisierung der Gattung, zur Phylogenie und zur vorgeschlagenen Klassifikation gemacht. Ein Schlüssel für die ơ nach äußeren Merkmalen beschließt das Kapitel, die Abbildungen dazu sind auf 9 Farbtafeln zusammengestellt. Die Daten zu diesen abgebildeten Faltern sind in Appendix 3 zusammengefaßt. Es folgen Bemerkungen zur Biogeografie und eine komprimierte Darstellung der gegenwärtigen Kenntnisse zur Biologie, Ökologie und Morphologie der Imagines und der Entwicklungsstadien. Die einzelnen Artengruppen werden charakteriasiert, es folgen Tafeln mit der Darstellung der ơ und \& Genitalien. Verbreitungskarten und das Literaturverzeichnis beschließen die Bearbeitung. Sehr nützlich für die Benutzung der bisher über diese Gattung erschienene Literatur ist Appendix 1, in dem die Fehlbestimmungen in der gängigen Literatur zwischen 1886 und 1997 aufgelistet sind.

Mit dieser monografischen Bearbeitung wurde eine verläßliche Grundlage für die weitere Erforschung dieser Lepidopterengruppe geschaffen. 